\title{
Effect of concurrent training on trainability performance factors in youth elite golf players
}

\author{
Juan Carlos Redondo ${ }^{\text {Corresp., } 1}{ }^{1}$, Ana María de Benito ${ }^{2}$, José María Izquierdo ${ }^{1}$ \\ 1 Department of Physical Education and Sports, University of León, León, Spain \\ 2 Physical Activity and Sports Sciences Faculty, Catholic University of Valencia San Vicente Mártir, Valencia, Spain \\ Corresponding Author: Juan Carlos Redondo \\ Email address: jc.castan@unileon.es
}

Background: Due to the early specialization of golf players, examining the within session sequence of training should be considered to enhance performance and prevent injury risk. The present study analyzed the effects of an 18-week concurrent training developed before or after a specific golf session in adolescence elite golfers on several performance factors.

Methods: Sixteen right-handed male golfers, were randomly divided into two groups: after golf specific training (AG) ( $n=8$, age: $16.77 \pm 0.58$ years) and before golf specific training $(B G)(n=8$, age: $16.93 \pm 0.59$ years). AG and BG players followed a concurrent physical conditioning program (CT) after or before the golf specific training, respectively. Body mass, body fat, muscle mass, jumping ability (CMJ), ball speed (Sball), golf movements screens (GMS), power in a golf swing-specific cable woodchop (Wmax) and the perceived training load (TL) in golf specific training (TL-G) and TL in CT (TL-CT) were measured on three separate occasions.

Results: BG demonstrates a lower TL-CT than AG $\left(p<.001, \eta_{p}{ }^{2}=0.90\right)$ along the training program without effects on TL-G, achieving significant percentage of change on CMJ $(9.38 \% ; p=.165 ; d=0.73)$, GMS (50.52\%; $\mathrm{p}=.41, d=0.91)$, Wmax (16.93\%; $\mathrm{p}=.001 ; d=2.02)$ and Sball $(1.82 \% ; \mathrm{p}=.018 ; d=$ 0.92 ) without interaction effects on anthropometric measures.

Conclusions: Performing CT sessions before the regular golf training can improve specific performance factors with a lower perceived TL than the same training carried out after the regular golf training. 


\section{Effect of concurrent training on trainability}

3 performance factors in youth elite golf players

4

5

6

7

8

9

10

11

12

13

14

15

16

17

18

19

20

21

22

23

24

25

26

27

28

29

30

31

32

33

34

35

36

37

Juan Carlos Redondo ${ }^{1}$

Ana María De Benito ${ }^{2}$

José María Izquierdo ${ }^{1}$

${ }^{1}$ Department of Physical Education and Sports, University of León, León, Spain.

${ }^{2}$ Physical Activity and Sports Sciences Faculty, Catholic University of Valencia San Vicente Mártir, Valencia, Spain.

Corresponding Author:

Juan Carlos Redondo ${ }^{1}$

Campus de Vegazana, S/N. León, 24071, Spain.

Email address: jc.castan@unileon.es

\section{Abstract}

Background: Due to the early specialization of golf players, examining the within session sequence of training should be considered to enhance performance and prevent injury risk. The present study analyzed the effects of an 18-week concurrent training developed before or after a specific golf session in adolescence elite golfers on several performance factors.

Methods: Sixteen right-handed male golfers, were randomly divided into two groups: after golf specific training $(A G)(n=8$, age: $16.77 \pm 0.58$ years $)$ and before golf specific training $(B G)(n=$ 8 , age: $16.93 \pm 0.59$ years). AG and BG players followed a concurrent physical conditioning program (CT) after or before the golf specific training, respectively. Body mass, body fat, muscle mass, jumping ability (CMJ), ball speed (Sball), golf movements screens (GMS), power in a golf swing-specific cable woodchop (Wmax) and the perceived training load (TL) in golf specific training (TL-G) and TL in CT (TL-CT) were measured on three separate occasions.

Results: BG demonstrates a lower TL-CT than AG $\left(\mathrm{p}<.001, \eta_{\mathrm{p}}{ }^{2}=0.90\right)$ along the training program without effects on TL-G, achieving significant percentage of change on CMJ $(9.38 \% ; p$ $=.165 ; d=0.73), \operatorname{GMS}(50.52 \% ; \mathrm{p}=.41, d=0.91), \operatorname{Wmax}(16.93 \% ; \mathrm{p}=.001 ; d=2.02)$ and Sball $(1.82 \% ; \mathrm{p}=.018 ; d=0.92)$ without interaction effects on anthropometric measures. Conclusions: Performing CT sessions before the regular golf training can improve specific performance factors with a lower perceived TL than the same training carried out after the regular golf training.

\section{Introduction}


Golf is a skill-based sport (Smith et al., 2011) but also a demanding physical game with high power requirements (Wells, Elmi, \& Thomas, 2009). Thus, golf practitioners need an adequate physical condition, where combined strength training is deemed necessary to golf performance (Doan et al., 2006; Thompson \& Osness, 2004).

In accordance with Lloyd et al. (2015a), golf players begin to specialize at the end of adolescence period and very few of them become elite professional players. Therefore, the sport talent development is a core aspect both athletes and practitioners. Enhancing youth golfers' performance is a complex and dynamic issue due to the varying interactions of growth, maturation, and training (Lloyd et al., 2014). For that reason, it is essential to design training strategies to optimize physical fitness and individual training response or trainability (Hecksteden et al., 2015). It's necessary to prescribe an accurate dosage of training load to prevent fatigue through training sessions and reduce injury risk. Myer et al. (2011) suggested that neuromuscular training is an effective method to prevent injuries in athletes when young.

In this sense, some studies conducted in youth golfers clarify the effects of strength training programs on physical fitness (Alvarez et al., 2012; Lamberth et al., 2013), but none has examined the within session sequence of neuromuscular training and sport-specific training as FernandezFernandez et al. (2018) conducted in youth tennis players. These authors based their work on Leveritt et al. (1999) who concluded that an acute bout of high-intensity endurance exercise may inhibit performance in a subsequent bout of resistance activity.

To the author's knowledge, there appears to be a lack of studies investigating the effects of different concurrent training on golf performance. Therefore, the purpose of this study was to assess the effects of an 18-week concurrent training developed before or after a specific golf session in elite adolescent golfers on several performance factors. We hypothesized that a concurrent training conducted before the specific golf training session would demonstrate greater increases on performance factors than the same concurrent training conducted after the specific golf training session.

\section{Materials \& Methods}

Study Design

A parallel, 2-group, longitudinal study was designed to investigate the effects of two different approaches of training on selected golf performance factors. Selected subjects had similar handicap to avoid golf swing technical differences. We assigned volunteers to either a training group conducting a concurrent physical conditioning program (CT) before golf specific training (BG) or a group that performed CT after golf specific training (AG). After a familiarization period, laboratory tests, and a specific range of physical- performance, participants were evaluated on three occasions; 1 week before the start of the training program (T1), after 12 weeks of training (T2) and after 18 weeks of training (T3). Also, subjects reported to be free from any injuries, surgeries or sport related rehabilitation during the 12 months prior to starting the study. The flowchart for recruitment and testing is displayed in Fig. 1.

Fig. 1. About Here 
79 The research was conducted during the competitive season (i.e., February, March, April, May

80 and June). Two months before the beginning of the study participants conducted the same

81 regular golf training program. Participants were instructed for not alter their lifestyle during the

82 investigation period in order to reduce potential interference. They were not allowed to exercise

83 or consume stimulant drinks at least 24 hours prior to test.

84 Participants

85 Based on the previous study by Alvarez et al. (2012) a priori power analysis (G*Power3) with

$86 \alpha<0.05$ and $1-\beta=80$ indicated that a sample size of at least 14 was required to explore the

87 differences between sequencing effects of neuromuscular training. A total of 16 elite right-

88 handed youth male golfers voluntarily agreed to participate in the study and were randomly

89 divided into two groups: before golf specific training $(B G, n=8)$ and after golf specific training

$90(\mathrm{AG}, \mathrm{n}=8)$. There were no group differences $(\mathrm{p}>.05)$ with regard to demographic and

91 anthropometric data showed in Table 1. Players averaged $9.4 \pm 0.9$ hours of training per week

92 and completed at least one full round of golf per week.

93 All players involved in the study attended all the sessions. Legal guardians and all participants

94 were provided an explanation of testing and training protocols and they gave written informed

95 consent prior to data collection. They also completed a set of questionnaires on their health

96 history and golf-playing history. This study was conducted in accordance with the guidelines

97 found in the Declaration of Helsinki and all procedures involving human subjects were approved

98 by the University of León Ethics Committee (ULE2018-2019-76).

99

100

101

102

103

104

105

106

107

108

109

110

111

112

113

114

115

116

117

118

\section{Testing procedures}

Table 1. About here

\section{Anthropometric Data.}

Anthropometric testing followed the International Society for the Advancement of

Kinanthropometry protocols (ISAK) (16 Fat mass, residual mass, bone mass, and muscle mass

and their respective percentages were computed to estimate body composition (Deurenberg et al. 1991).

In order to estimate the maturity status of participants, the peak-height-velocity (PHV) was calculated according to Mirwald et al. (2002). All anthropometric measures were highly reliable with intraclass correlation coefficients (ICCs) of 0.91 to 0.98 for skinfolds and 0.93 to 0.98 for diameters.

Training Load Quantification.

During 18 in-season weeks, the perceived training load (TL) was quantified using the session rating of perceived exertion (sRPE) method (Foster et al. 2001). Ten minutes after each training and using Foster's 0-10 scale (Foster et al. 2001), participants were asked by the same person (fitness coach) on all occasions to rate their general perception of the session difficulty (PE) (Chen et al. 2002). We allowed players to mark a plus sign (interpreted as 0.5 points) alongside the integer value (Otaegi \& Los Arcos 2020). All the golfers were familiarized with this method during the previous months. All golf specific training and CT PEs recorded during the study were summed separately. Then, TL was calculated by multiplying the PE value by the duration 
119 of the training. Partial 12-weeks (T2) and 18-weeks (T3) TL in golf specific training (TL-G) and 120 TL in CT (TL-CT) were considered for each group (Otaegi \& Los Arcos 2020). The duration of 121 a training session (training volume) was recorded for each player from the start to the end of the 122 session, including recovery periods but excluding stretching exercises (Los Arcos et al. 2015).

123 Golf Movement Screen

124 We applied a specific golf movement screen (GMS) to examine the movement competency of golf players. According to Gulgin et al. (2014), the subjects performed 13 different tests (movement screens). These tests, established by the Titleist Performance Institute (TPI), provide data with respect to stability, mobility, coordination of body segments, and balance. The sum of the 13 GMS was recorded. The ICC was 0.98 . Lower Limbs Explosive Strength Golfers performed a countermovement jump (CMJ) without arm swing on a jumping mat (SportJUMP System; DSD, Spain) according to Bosco et al. (1983). Golfers performed two maximal CMJs intercalated with $60 \mathrm{~s}$ of passive recovery. Only the best height for each participant was recorded. The ICC of the CMJ was 0.97 and the CV was $4.1 \%$. Rotational Golf-specific Exercise According to Keogh et al. (2009) the golf swing-specific cable woodchop (GSCWC) is a rotational exercise that is very similar to the golf swing in terms of posture, range of motion, intended velocity, direction of force (torque) application, and coordination patterns. A one-repetition-maximum (1-RM) test following the protocol established by the National Strength and Conditioning Association was performed to measure peak power output (highest instantaneous value) during each GSCWC exercise. The peak power outputs (Wmax) expressed in watts were measured with a pneumatic resistance device (Infinity, Keiser, Calif. USA) according to Peltonen et al. (2013). The ICC was 0.99. Driving Performance.

144 Ball speed (Sball) was assessed using new regulation golf balls (Titlest Pro V1, USA), and tees of various heights to suit the preference of each participant. According to Alvarez et al. (2012), Sball expressed in $\mathrm{km} \cdot \mathrm{h}-1$ was measured with a Stalker's type hyperfrequency radar (Stalker Professional Radar, Radar Sales, Plymouth, MA, USA). Each participant performed five drives at the maximum speed possible using his own club. The ICC for this test was 0.94 . Training intervention sessions (on Wednesday and Friday), one putter-and-approach session on Monday and one full round of golf (on Saturday or Sunday). neuromuscular training program (Table 2) was undertaken at an indoor facility (high performance sports center) on average $66.70 \pm 3.1$ minutes per session. The regular golf training took place at an outdoor facility (sport golf club center) located 30 minutes from each other.

157 According to Fernandez-Fernandez et al. (2018) during recovery period between training bouts, 158 all participants could ingest water and carbohydrate/electrolyte drink. Regular golf training lasted 
159

160

161

162

163

164

165

166

167

168

169

170

171

172

173

174

175

176

177

178

179

180

181

182

183

184

185

186

187

188

189

190

191

192

193

194

195

196

on average $83.2 \pm 9.6$ minutes and was characterized by a $\sim 10$-minute specific warm-up (i.e., general mobility and low-intensity golf shots), $\sim 30$ minutes of technical swing adjustments, and $\sim 40$ minutes of specific drills (i.e., mixed iron-drives-putter drills).

All players had previous experience of this type of training. Prior to starting CT, participants performed a standardized 10-minute warm-up protocol. After the warm-up, each golfer developed a 30-minutes personal golf-specific functional movement training program with conditioning exercises designed to enhance the lower body stability and the upper body mobility (Lephart et al. 2007). Lastly, participants proceeded with the neuromuscular training program divided into three parts (maximal strength; explosive strength and golf-specific strength training), each six weeks long (Alvarez et al. 2012). Details are given in Table 2.

Table 2. About here

\section{Data analyses.}

The data were checked for normality using the Shapiro-Wilk test and found to be suitable for parametric testing. Student's t-tests were performed to determine differences between groups at baseline. A $2 \times 3$ repeated measures ANOVA was used to explore the effects of group (AG and $\mathrm{BG}$ ), and time (one week before training, 12 weeks and 18 weeks after training). When a significant $F$ value was achieved by means of Wilks' lambda, Scheffe's post hoc procedures were performed to locate the pairwise differences. In addition, partial eta squared $\left(\eta_{\mathrm{p}}{ }^{2}\right)$ was computed to determine the effect size which was interpreted as small 0.1 , medium 0.3 , and large 0.5 . The percentage difference between groups was assessed using one-way ANOVA by comparing T1-T2, T1-T3 and T2-T3 and the Cohen's $d$ (Cohen, 1988) was calculated to determine the magnitude of differences between experimental conditions for each variable. The significance level was set at $\mathrm{p} \leq .05$. Statistical analysis was performed with SPSS 24.0 (IBM ${ }^{\circledR}$ SPSS Statistics 24, IBM GmbH).

\section{Results}

Overall, golfers completed more than $95 \%$ of the training sessions, proving a very good adhesion to the training program. Student's t-test between AG and BG at baseline reveled that there were no statistically significant differences $(p>.05)$ before the start of the training program with regard to the analyzed variables. The mean and standard deviation and main effects for the different variables are reported in Table 3 and Table 4.

Anthropometric Data

ANOVA revealed no significant time $\mathrm{x}$ group interaction for anthropometric measures, although significant improvements were seen between the time points for both groups. Further post hoc analysis showed significant increase of body mass between T1 and T2 $(\mathrm{p}<.001 ; d=0.14), \mathrm{T} 2$ and $\mathrm{T} 3(\mathrm{p}=.03 ; d=0.04)$ and $\mathrm{T} 1$ and $\mathrm{T} 3(\mathrm{p}=.002 ; d=0.18)$ in $\mathrm{BG}$, and between $\mathrm{T} 2$ and $\mathrm{T} 3(\mathrm{p}=.04$; $d=0.06)$ in AG. Related to percent muscle mass, a significant increase was observed in BG between $\mathrm{T} 1$ and $\mathrm{T} 3(\mathrm{p}=.04 ; d=0.34)$.

Perceived Training Load 
197 Data analysis revealed significant time $\mathrm{x}$ group interaction effects just for TL-CT $\left(\mathrm{p}=.005 ; \eta_{\mathrm{p}}{ }^{2}=\right.$ 198 0.45). Differences between T2 and T3 were dismissed as non-logic (neuromuscular training load 199 in T2 not comparable with golf-specific training load in T3).

200 Performance variables

201 Analysis of variance located significant time $\mathrm{x}$ group interaction effects for GMS $\left(\mathrm{p}=.02 ; \eta_{\mathrm{p}}{ }^{2}=\right.$ $2020.25)$ and $W \max \left(p<.001 ; \eta_{\mathrm{p}}{ }^{2}=0.53\right)$. Additionally, ANOVA revealed a significant effect for 203 time in all the performance variables. Regarding to BG, Scheffe's post hoc tests located the 204 differences between T1 and T2 differences were located in GMS $(\mathrm{p}<.002 ; d=1.06)$, CMJ (p $<$ $205.001 ; d=0.34)$, Wmax $(\mathrm{p}<.001 ; d=0.67)$ and Sball $(\mathrm{p}<.01 ; d=0.24)$, between $\mathrm{T} 1$ and T3 in 206 GMS $(\mathrm{p}<.001 ; d=1.81)$, CMJ $(\mathrm{p}<.001 ; d=0.78)$, Wmax $(\mathrm{p}<.001 ; d=1.21)$ and Sball $(\mathrm{p}<$ 207

208

209

210

211

212

213 $.001 ; d=0.31)$, and between T2 and T3 differences were located in GMS ( $\mathrm{p}=.003 ; d=0.97)$, $\mathrm{CMJ}(\mathrm{p}=.03 ; d=0.44)$ and Wmax $(\mathrm{p}<.001 ; d=0.56)$. Furthermore, post hoc analysis for AG located the differences between T1 and T2 differences were located in CMJ $(\mathrm{p}<.001 ; d=0.40)$ and Wmax $(\mathrm{p}<.001 ; d=0.22)$, between T1 and T3 in CMJ $(\mathrm{p}=.02 ; d=0.43)$, Wmax $(\mathrm{p}=.002$; $d=0.41)$ and Sball $(\mathrm{p}=.02 ; d=0.34)$, and between T2 and T3 differences were located in CMJ $(\mathrm{p}=.02 ; d=0.03)$ and $\mathrm{Wmax}(\mathrm{p}=.03 ; d=0.18)$.

214 With regard to comparison of the percentage of change between evaluations (T1, T2 and T3) in association with the TL data are represented in Fig. 2. Concerning TL-CT, one-way ANOVA

215 revealed a significant effect between $\mathrm{AG}$ and $\mathrm{BG}$ in T2 $(\mathrm{p}<.001 ; d=3.43)$ and $\mathrm{T} 3(\mathrm{p}=.018 ; d=$ 216 1.34). T1-T3 comparison between groups shows that $\mathrm{BG}$ obtains higher percentages of change in all performance variables: $\mathrm{CMJ}(\mathrm{AG}+37.15 \%$; $\mathrm{BG}+50.52 \% ; \mathrm{p}=.041 ; d=0.91)$, GMS (AG $+5.08 \% ; \mathrm{BG}+9.38 \% ; \mathrm{p}=.165 ; d=0.73), \mathrm{Wmax}(\mathrm{AG}+8.03 \% ; \mathrm{BG}+16.96 \% ; \mathrm{p}=.001 ; d=2.02)$

219

220

221

222

223 and Sball $(\mathrm{AG}+1.03 \% ; \mathrm{BG}+1.82 \% ; \mathrm{p}=.018 ; d=0.92)$.

Table 3. About here

Table 4. About here

Fig. 2. About here

224

225

226

227

228

229

230

231

232

233

234

235

236

\section{Discussion}

The purpose of the current investigation included comparing the effects of an 18-week concurrent training developed before or after a specific golf session in adolescent elite golfers on several performance factors. Key findings for the sequencing effects of training programs showed that CT conducted before the specific golf training demonstrate greater increases on performance factors and less perceived training load than the same CT conducted after the specific golf training.

Experts have considered the use of session RPE needless for on and off-course golf activities and it would seem that rejection is based on a perceived low intensity of golf (Williams et al., 2018). However, such considerations have not been raised into the context of routinely training programs of golf players. In regular golf training, BG and AG were requested to execute a wide range of skills that require both fine motor skills and muscular power over an extended period of time (Hellström, 2009). In this context, TL-G outcomes showed no differences for BG compared to

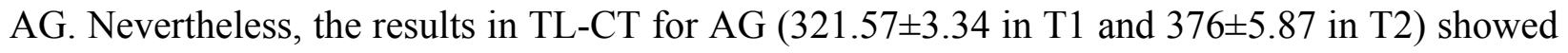




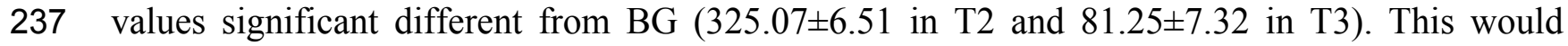
238 indicate that golfers in the $\mathrm{BG}$ were carrying out the $\mathrm{CT}$ sessions in a less sense of fatigue, resulting 239 in greater increases on performance factors possibly due to a less stress (Blume et al., 2018).

240 Improvements obtained in CMJ (5.08\% for AG and 9.38\% for BG) are agree with previous studies 241 (Alvarez et al., 2012; Driggers \& Sato, 2018; Kenny et al., 2017), confirming that a twice-weekly 242 strength training program, using the protocol outlined, was associated with enhancements in 243 driving performance (Wells et al., 2019), lower limb explosive strength and rotational power. Our 244 results revealed that golfers transfer the gains to the driver performance with percentage 245 improvement ranging from $1.03 \%$ to $1.82 \%$ for $\mathrm{AG}$ and $\mathrm{BG}$ respectively.

246 Previous studies have reported the positive influence of strength training on driving performance 247 in highly-trained players (Alvarez et al., 2012; Driggers \& Sato, 2018; Fletcher \& Hartwell, 2004).

248 This is confirmed by the results of our study, which proves that the CT program followed increase 249

250

251

252

253

254

255

256

257 driving performance, both Sball and rotational power. Our results show that BG obtains higher improvements than AG in GMS (50.52\% vs $37.15 \%)$ and rotational power (16.96\% vs $8.03 \%)$ while maintaining similar differences in Sball. The enhancements in driving performance could be related to improvements in GMS such as stability, mobility, body segment coordination and balance (Gulgin et al., 2014; Myers et al., 2008; Speariett \& Armstrong, 2019). Further 3D motion analysis work would provide deeper analysis to clarify the relation between swing mechanics and strength and golf movement screens.

258 Golf can be one of those sports traditionally favor early specialization (Lloyd et al., 2015b) for this reason golfers should be engaged with an integrative strength and conditioning programs focused on diversifying motor skill development and enhancing muscle strength to maximize performance

259 and reduce injury risk (Faigenbaum et al., 2014). In this regard, our data show that concurrent 260

261 training programs combining physical conditioning program and golf specific training may have an important impact on performance factors such as drive ball speed (Torres-Ronda et al., 2014)

262

263 or CMJ (Driggers \& Sato, 2018; Kenny et al., 2017). In addition, session sequence should be considered since our results suggest that performing strength training before golf-specific work 265

266 allows golfers get a better or similar performance with a lower perceived TL ( $9 \%$ lower BG than AG) which supports the results of $18 \%$ obtained by Fernandez-Fernandez et al. (2019).

267 On the basis of our results, it may be concluded that implement concurrent training before a 268 specific-golf session in young golf players is more effective over golf performance factors (e.g. 269 jumping performance, ball speed, rotation power). Thus, coaches would develop combined golf270 specific functional movement (e.g. Titleist Performance Institute, level 1 golf fitness screen) and 271 neuromuscular training program divided into three parts (maximal, explosive and golf-specific 272 strength). From a practical point of view, CT sessions should not exceed a total volume of 70 273 min (including the warm- up) and an appropriate resting time before the following golf training 274 should be above 30 min.

\section{References}


276 Alvarez M, Sedano S, Cuadrado G, and Redondo JC. 2012. Effects of an 18-week strength

277 training program on low-handicap golfers' performance. J Strength Cond Res 26:1110-1121.

278 10.1519/JSC.0b013e31822dfa7d

279 Blume K, Körber N, Hoffmann D, and Wolfarth B. 2018. Training Load, Immune Status, and

280 Clinical Outcomes in Young Athletes: A Controlled, Prospective, Longitudinal Study. Frontiers

281 in Physiology 9. 10.3389/fphys.2018.00120

282 Bosco C, Luhtanen P, and Komi PV. 1983. A simple method for measurement of mechanical

283 power in jumping. Eur J App Physiol Occup Physiol 50:273-282.

284 Chen MJ, Fan X, and Moe ST. 2002. Criterion-related validity of the Borg ratings of perceived

285 exertion scale in healthy individuals: a meta-analysis. J Sports Sci 20:873-899.

286 Cohen J. 1988. Statistical power analysis for the behavioral -Second Edition. 12 Lawrence

287 Erlbaum Associates Inc. Hillsdale, New Jersey, 13.

288 Deurenberg P, Weststrate JA, and Seidell JC. 1991. Body mass index as a measure of body

289 fatness: age-and sex-specific prediction formulas. Br J Nutr 65:105-114.

290 Doan BK, Newton RU, Kwon Y-h, and Kraemer WJ. 2006. Effects of physical conditioning on 291 intercollegiate golfer performance. J Strength Cond Res 20:62-72.

292 Driggers AR, and Sato K. 2018. The effects of vertically oriented resistance training on golf 293 drive performance in collegiate golfers. Int J Sports Sci Coach 13:598-606.

294 https://doi.org/10.1177/1747954117743374

295 Faigenbaum AD, Myer GD, Farrell A, Radler T, Fabiano M, Kang J, Ratamess N, Khoury J, and 296 Hewett TE. 2014. Integrative neuromuscular training and sex-specific fitness performance in 7297 year-old children: an exploratory investigation. J Athl Train 49:145-153.

298 https://doi.org/10.4085/1062-6050-49.1.08

299 Farrally M, Cochran A, Crews D, Hurdzan M, Price R, Snow J, and Thomas P. 2003. Golf

300 science research at the beginning of the twenty-first century. J Sport Sci 21:753-765.

301 https://doi.org/10.1080/0264041031000102123

302 Fernandez-Fernandez J, Granacher U, Sanz-Rivas D, Marín JMS, Hernandez-Davo JL, and 303 Moya M. 2018. Sequencing Effects of Neuromuscular Training on Physical Fitness in Youth 304 Elite Tennis Players. J Strength Cond Res 32:849-856. 10.1519/JSC.0000000000002319

305 Fletcher IM, and Hartwell M. 2004. Effect of an 8-week combined weights and plyometrics 306 training program on golf drive performance. J Strength Cond Res 18:59-62.

307 Foster C, Florhaug JA, Franklin J, Gottschall L, Hrovatin LA, Parker S, Doleshal P, and Dodge 308 C. 2001. A new approach to monitoring exercise training. J Strength Cond Res 15:109-115. 309 Gulgin HR, Schulte BC, and Crawley AA. 2014. Correlation of Titleist Performance Institute 310 (TPI) level 1 movement screens and golf swing faults. J Strength Cond Res 28:534-539. 311 10.1519/JSC.0b013e31829b2ac4

312 Hecksteden A, Kraushaar J, Scharhag-Rosenberger F, Theisen D, Senn S, and Meyer T. 2015. 313 Individual response to exercise training-a statistical perspective. J Appl Physiol 118:1450-1459. 314 https://doi.org/10.1152/japplphysiol.00714.2014 
315 Hellström J. 2009. Competitive elite golf. Sports Med 39:723-741.

316 https://doi.org/10.2165/11315200-000000000-00000

317 Kenny D-M, Presnall J, Cosio-Lima L, and Greska E. 2017. The effects of a 5-week golf specific

318 strength and conditioning intervention on swing performance factors. Br J Sports Med 51:339-

319 339. http://dx.doi.org/10.1136/bjsports-2016-097372.142

320 Keogh JW, Marnewick MC, Maulder PS, Nortje JP, Hume PA, and Bradshaw EJ. 2009. Are

321 anthropometric, flexibility, muscular strength, and endurance variables related to clubhead

322 velocity in low-and high-handicap golfers? J Strength Cond Res 23:1841-1850.

323 10.1519/JSC.0b013e3181b73cb3

324 Lamberth J, Hale B, Knight A, Boyd J, and Luczak T. 2013. Effectiveness of a six-week strength 325 and functional training program on golf performance. International Journal of Golf Science 2:3332642.

327 Lephart SM, Smoliga JM, Myers JB, Sell TC, and Tsai Y-s. 2007. An eight-week golf-specific

328

329

330

331

332

333

334

335

336

337

338

339

340

341

342

343

344

345

346

347

348

349

350

351

352 exercise program improves physical characteristics, swing mechanics, and golf performance in recreational golfers. J Strength Cond Res 21:860-869.

Lloyd RS, Oliver JL, Faigenbaum AD, Howard R, Croix MBDS, Williams CA, Best TM, Alvar BA, Micheli LJ, and Thomas DP. 2015a. Long-term athletic development-part 1: a pathway for all youth. J Strength Cond Res 29:1439-1450. 10.1519/JSC.0000000000000756

Lloyd RS, Oliver JL, Faigenbaum AD, Howard R, De Ste Croix MBA, Williams CA, Best TM, Alvar BA, Micheli LJ, Thomas DP, Hatfield DL, Cronin JB, and Myer GD. 2015b. Long-Term Athletic Development, Part 2: Barriers to Success and Potential Solutions. J Strength Cond Res 29:1451-1464. 10.1519/01.jsc.0000465424.75389.56

Lloyd RS, Oliver JL, Faigenbaum AD, Myer GD, and Croix MBDS. 2014. Chronological age vs. biological maturation: implications for exercise programming in youth. J Strength Cond Res 28:1454-1464. 10.1519/JSC.0000000000000391

Los Arcos A, Martínez-Santos R, Yanci J, Mendiguchia J, and Méndez-Villanueva A. 2015.

Negative associations between perceived training load, volume and changes in physical fitness in professional soccer players. J Sports Sci Med 14:394.

Mirwald RL, Baxter-Jones AD, Bailey DA, and Beunen GP. 2002. An assessment of maturity from anthropometric measurements. Med Sci Sports Exerc 34:689-694.

Myers J, Lephart S, Tsai Y-s, Sell T, Smoliga J, and Jolly J. 2008. The role of upper torso and pelvis rotation in driving performance during the golf swing. J Sport Sci 26:181-188.

https://doi.org/10.1080/02640410701373543

Otaegi A, and Los Arcos A. 2020. Quantification of the perceived training load in young female basketball players. J Strength Cond Res 34:559-565. https://doi.org/10.1519/jsc.0000000000002370

Speariett S, and Armstrong R. 2019. The Relationship Between the Golf-Specific Movement Screen and Golf Performance. J Sports Rehabil 1:1-11. https://doi.org/10.1123/jsr.2018-0441 
353 Torres-Ronda L, Delextrat A, and Gonzalez-Badillo JJ. 2014. The relationship between golf

354 performance, anthropometrics, muscular strength and power characteristics in young elite 355 players. International SportMed Journal 15:156-164.

356 Williams SB, Gastin PB, Saw AE, and Robertson S. 2018. Development of a golf-specific load

357 monitoring tool: Content validity and feasibility. Eur J Sport Sci 18:458-472.

$358 \quad 10.1080 / 17461391.2018 .1434239$ 


\section{Table 1 (on next page)}

Demographic and anthropometric data of the players (mean \pm SD)

$A G=$ after golf specific training, $B G=$ before golf specific training, $P H V=$ peak height velocity 


\begin{tabular}{cccccccc}
\hline Group & $\mathbf{n}$ & $\begin{array}{c}\text { Age } \\
(\mathbf{y})\end{array}$ & $\begin{array}{c}\text { Experience } \\
(\mathbf{y})\end{array}$ & Handicap & $\begin{array}{c}\text { Height } \\
(\mathbf{c m})\end{array}$ & $\begin{array}{c}\text { Mass } \\
(\mathbf{k g})\end{array}$ & PHV \\
\hline AG & 8 & $16.44 \pm 0.67$ & $3.1 \pm 1.2$ & $0.24 \pm 0.79$ & $176.14 \pm 6.98$ & $71.56 \pm 7.81$ & $1.84 \pm 0.70$ \\
BG & 8 & $16.28 \pm 0.58$ & $3.0 \pm 0.9$ & $0.34 \pm 1.21$ & $176.28 \pm 4.08$ & $67.38 \pm 12.41$ & $1.53 \pm 0.80$ \\
\hline
\end{tabular}

1 
Table 2 (on next page)

Neuromuscular training details 


\begin{tabular}{|c|c|}
\hline \multicolumn{2}{|l|}{ Maximal Strength Training } \\
\hline Resistance Exercise & Sets/Repetitions/Load/Rest Period Between Sets \\
\hline $\begin{array}{l}\text { Horizontal bench press } \\
\text { Seated row machine } \\
\text { Leg press machine } \\
\text { Seated calf extension } \\
\text { Triceps cable push-down } \\
\end{array}$ & $\begin{array}{l}3 \text { sets } \times 5 \text { repetitions } \times 80 \% / 4 \text { minutes } \\
3 \text { sets } \times 5 \text { repetitions } \times 80 \% / 4 \text { minutes } \\
3 \text { sets } \times 5 \text { repetitions } \times 80 \% / 4 \text { minutes } \\
3 \text { sets } \times 5 \text { repetitions } \times 80 \% / 4 \text { minutes } \\
3 \text { sets } \times 5 \text { repetitions } \times 80 \% / 4 \text { minutes }\end{array}$ \\
\hline \multicolumn{2}{|l|}{ Explosive Strength Training } \\
\hline Combined exercise & Sets/Repetitions/Load/Repetitions/Rest Between Sets \\
\hline $\begin{array}{l}\text { Horizontal bench press + plyometric push-ups } \\
\text { Seated row machine }+ \text { explosive pull-downs } \\
\text { Leg press machine + vertical jumps over } \\
\text { hurdles }(45 \mathrm{~cm})\end{array}$ & $\begin{array}{l}3 \text { sets }(6 \text { repetitions } \times 70 \%+10 \text { repetitions) } / 4 \text { minutes } \\
3 \text { sets }(6 \text { repetitions x } 70 \%+10 \text { repetitions }) / 4 \text { minutes } \\
3 \text { sets ( } 6 \text { repetitions } \times 70 \%+10 \text { repetitions) } / 4 \text { minutes }\end{array}$ \\
\hline $\begin{array}{l}\text { Seated calf extension }+ \text { vertical jumps over } \\
\text { hurdles }(45 \mathrm{~cm}) \\
\text { Triceps cable push-down }+ \text { plyometric } \\
\text { push-ups }\end{array}$ & $\begin{array}{l}3 \text { sets ( } 6 \text { repetitions x } 70 \%+10 \text { repetitions) } / 4 \text { minutes } \\
3 \text { sets ( } 6 \text { repetitions x } 70 \%+10 \text { repetitions) } / 4 \text { minutes }\end{array}$ \\
\hline \multicolumn{2}{|l|}{ Golf-Specific Strength Training } \\
\hline Exercises & Sets/Repetitions/Rest Between Sets \\
\hline $\begin{array}{l}\text { Golf drives with weighted clubs } \\
\text { Accelerated drives with an acceleration tubing } \\
\text { club system }\end{array}$ & $\begin{array}{l}3 \text { sets } \times 10 \text { repetitions } / 4 \text { minutes } \\
3 \text { sets } \times 10 \text { repetitions } / 4 \text { minutes }\end{array}$ \\
\hline
\end{tabular}




\section{Table 3 (on next page)}

Descriptive and inferential anthropometric results from 2 (group) $\times 3$ (time) ANOVA.

AG, after golf group; BG, before golf group; T1, 1 week before training program; T2, after 12

weeks of training; T3, after 18 weeks of training; $p$, p value; $\eta_{p}{ }^{2}$, effect size; $\dagger$, significant difference between T1 and T2; $\ddagger$, significant difference between T2 and T3. 


\begin{tabular}{|c|c|c|c|c|c|c|c|}
\hline \multirow{2}{*}{ Group/time } & & \multicolumn{2}{|c|}{ Body mass, kg } & \multicolumn{2}{|c|}{ Body fat percent } & \multicolumn{2}{|c|}{ Muscle mass percen } \\
\hline & & $\mathbf{M}$ & SD & $\mathbf{M}$ & SD & $\mathbf{M}$ & SD \\
\hline \multirow[t]{3}{*}{$\mathrm{AG}$} & T1 & 71.56 & 7.81 & 10.45 & 2.13 & 48.28 & 2.01 \\
\hline & $\mathrm{T} 2$ & $72.25 \ddagger$ & 7.31 & 10.37 & 2.01 & 48.53 & 1.97 \\
\hline & $\mathrm{T} 3$ & 72.71 & 7.71 & 10.11 & 2.13 & 48.68 & 1.98 \\
\hline \multirow[t]{3}{*}{ BG } & $\mathrm{T} 1$ & $67.38 \dagger$ & 12.41 & 11.42 & 2.58 & 46.87 & 1.94 \\
\hline & $\mathrm{T} 2$ & $69.01 \%$ & 11.86 & 11.55 & 2.24 & 47.18 & 1.84 \\
\hline & $\mathrm{T} 3$ & 69.51 & 11.77 & 11.01 & 2.11 & 47.47 & 1.63 \\
\hline RM ANOVA & & $p$ & $\eta_{p}^{2}$ & $p$ & $\eta_{p}^{2}$ & $p$ & $\eta_{p}^{2}$ \\
\hline Group & & .49 & 0.03 & .36 & 0.06 & .18 & 0.12 \\
\hline Time & & $<.001$ & 0.57 & .20 & 0.2 & .001 & 0.42 \\
\hline Time x Group & & .15 & 0.13 & .84 & 0.01 & .65 & 0.03 \\
\hline
\end{tabular}

1 


\section{Table 4 (on next page)}

Descriptive and inferential perceived training load and performance results from 2 (group) $\times 3$ (time) ANOVA.

n/a, not applicable; AG, after golf group; $B G$, before golf group; $T 1,1$ week before training program; T2, after 12 weeks of training; T3, after 18 weeks of training; $p, p$ value; $\eta_{p}{ }^{2}$, effect size; TL-CT, perceived concurrent physical conditioning training load; TL-G, perceived specific golf training load; au, arbitrary units; CMJ, countermovement jump; GMS, golf movement screen; Sball, ball speed; Wmax, maximal power; $\dagger$, significant difference between T1 and T2; $\ddagger$, significant difference between T2 and T3. 


\begin{tabular}{|c|c|c|c|c|c|c|c|c|c|c|c|c|c|}
\hline \multirow{2}{*}{ Group/time } & & \multicolumn{2}{|c|}{ TL-CT, au } & \multicolumn{2}{|c|}{ TL-G, au } & \multicolumn{2}{|c|}{ CMJ, cm } & \multicolumn{2}{|c|}{ GMS, au } & \multicolumn{2}{|c|}{ Sball, $\mathbf{k m} \cdot \mathbf{h}^{-1}$} & \multicolumn{2}{|c|}{ Wmax, w } \\
\hline & & $\mathbf{M}$ & SD & $\mathbf{M}$ & SD & $\mathbf{M}$ & SD & $\mathbf{M}$ & SD & $\mathbf{M}$ & SD & $\mathbf{M}$ & SD \\
\hline \multirow{2}{*}{$\mathrm{AG}$} & $\mathrm{T}$ & \multirow{2}{*}{\multicolumn{2}{|c|}{$\mathrm{n} / \mathrm{a}$}} & \multirow{2}{*}{\multicolumn{2}{|c|}{$\mathrm{n} / \mathrm{a}$}} & \multirow{2}{*}{$40.15 \dagger$} & \multirow{2}{*}{4.98} & \multirow{2}{*}{11.13} & 4.4 & \multirow{2}{*}{256.63} & \multirow{2}{*}{8.63} & \multirow{2}{*}{$965.13 \dagger$} & 204.5 \\
\hline & 1 & & & & & & & & 2 & & & & 7 \\
\hline \multirow{8}{*}{ BG } & $\begin{array}{l}\mathrm{T} \\
2\end{array}$ & 349.06 & 4.57 & $\begin{array}{c}209.2 \\
7\end{array}$ & 5.78 & $42.13 \%$ & 4.91 & 8.75 & $\begin{array}{c}5.7 \\
3\end{array}$ & 258.5 & 6.65 & 1010.88 & $\begin{array}{c}209.1 \\
9\end{array}$ \\
\hline & $\begin{array}{l}\mathrm{T} \\
3\end{array}$ & 90.63 & 6.65 & 210.0 & 10.34 & $42.29 \S$ & 4.88 & 7.63 & $\begin{array}{c}4.0 \\
7\end{array}$ & $259.5 \S$ & 6.85 & $1048.88 \S$ & $\begin{array}{c}208.4 \\
9\end{array}$ \\
\hline & $\mathrm{T}$ & $\mathrm{n}$ & & & & $37.07 \dagger$ & 5.11 & $13.5 \dagger$ & 5.7 & 250.88 & 15.3 & $992.75 \dagger$ & 164.5 \\
\hline & 1 & & & & & & & & 3 & $\dagger$ & 15.3 & | & 3 \\
\hline & $\mathrm{T}$ & 32507 & 651 & 211.4 & 517 & $3879 \div$ & 489 & $85 t$ & 3.4 & 2545 & 14.9 & $11020+$ & 160.9 \\
\hline & 2 & 523.01 & 0.01 & 6 & 0.11 & $50.19+$ & 4.09 & $0 . J_{t}$ & 6 & $204 . J$ & 7 & 1102.0 & 3 \\
\hline & $\mathrm{T}$ & 8125 & 732 & 213.6 & 1031 & 4088 & 464 & 588 & 1.6 & 25663 & 14.9 & 119350 & 168.3 \\
\hline & 3 & $01.2 J$ & 1.02 & 5 & 10.51 & 40.00 & 4.04 & 3.00 & 4 & 250.05 & 9 & ס & 2 \\
\hline RM ANOVA & & $p$ & $\eta_{p}^{2}$ & $p$ & $\eta_{p}^{2}$ & $p$ & $\eta_{p}^{2}$ & $p$ & $\eta_{p}^{2}$ & $p$ & $\eta_{p}^{2}$ & $p$ & $\eta_{p}^{2}$ \\
\hline Group & & $<.001$ & 0.79 & .46 & 0.04 & .30 & 0.08 & .16 & $\begin{array}{c}0.2 \\
5\end{array}$ & .45 & 0.04 & .36 & 0.06 \\
\hline Time & & $<.001$ & 0.99 & .26 & 0.19 & $<.001$ & 0.65 & $<.001$ & $\begin{array}{c}0.6 \\
4\end{array}$ & $<.001$ & 0.63 & $<.001$ & 0.87 \\
\hline Time x Group & & .005 & 0.45 & .86 & 0.02 & .06 & 0.18 & .02 & $\begin{array}{c}0.2 \\
5\end{array}$ & .15 & 0.13 & $<.001$ & 0.53 \\
\hline
\end{tabular}


Figure 1

Recruitment and testing flowchart of participants through the intervention

\section{Study FlowChart}

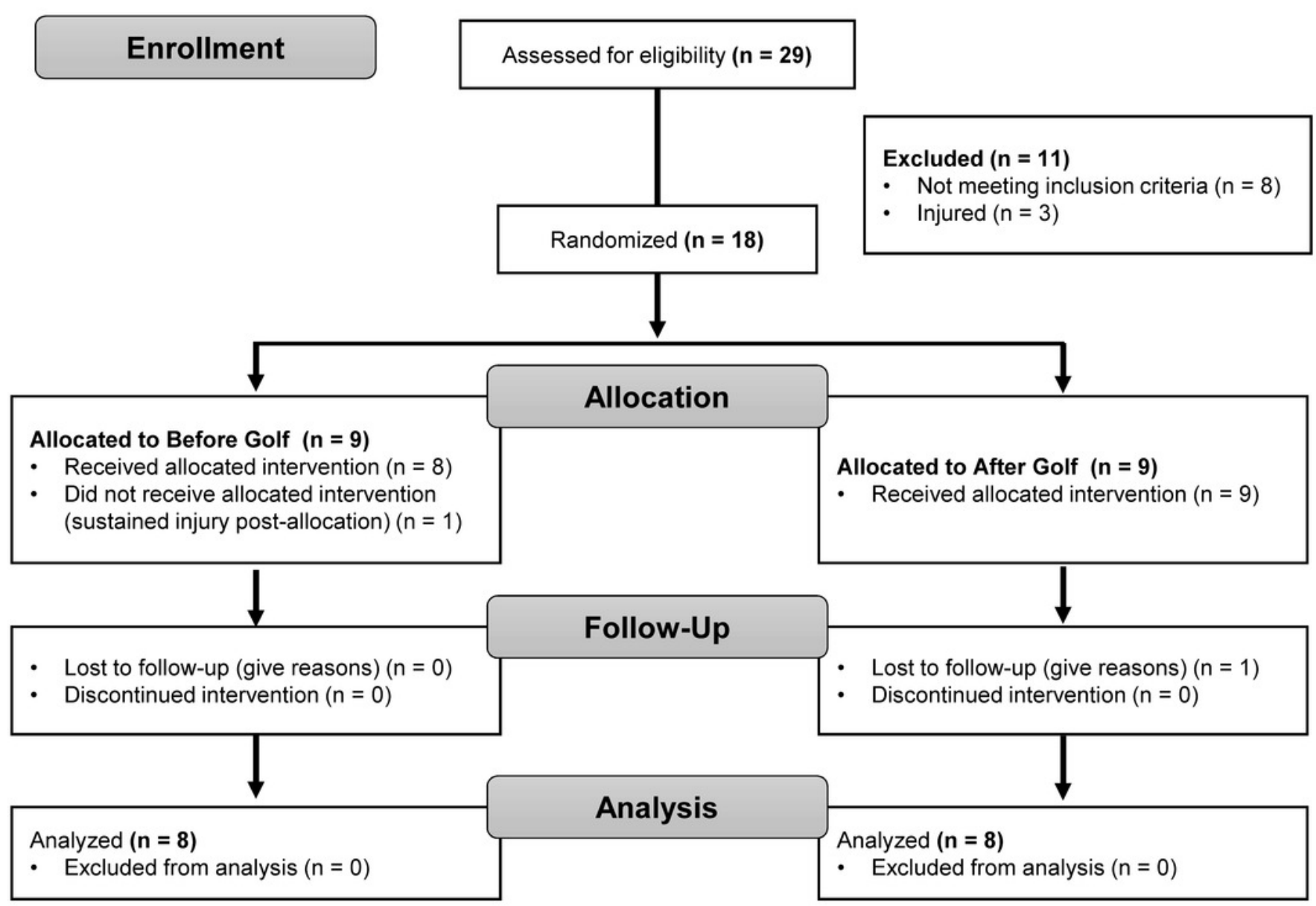




\section{Figure 2}

Comparison of the percentage of change between evaluations ( $\mathrm{T} 1, \mathrm{~T} 2$ and $\mathrm{T} 3$ ).

AG, after golf group; BG, before golf group; au, arbitrary units; (A) TL-CT, perceived concurrent physical conditioning training load; (B) TL-G, perceived specific golf training load; (C) CMJ, countermovement jump; (D) GMS, golf movement screen; (E) Sball, ball speed; (F) Wmax, maximal power. \# percentage of change larger in BG than in AG $(p<.001)$; * significance difference between $A G$ and $B G(p<.001)$. 


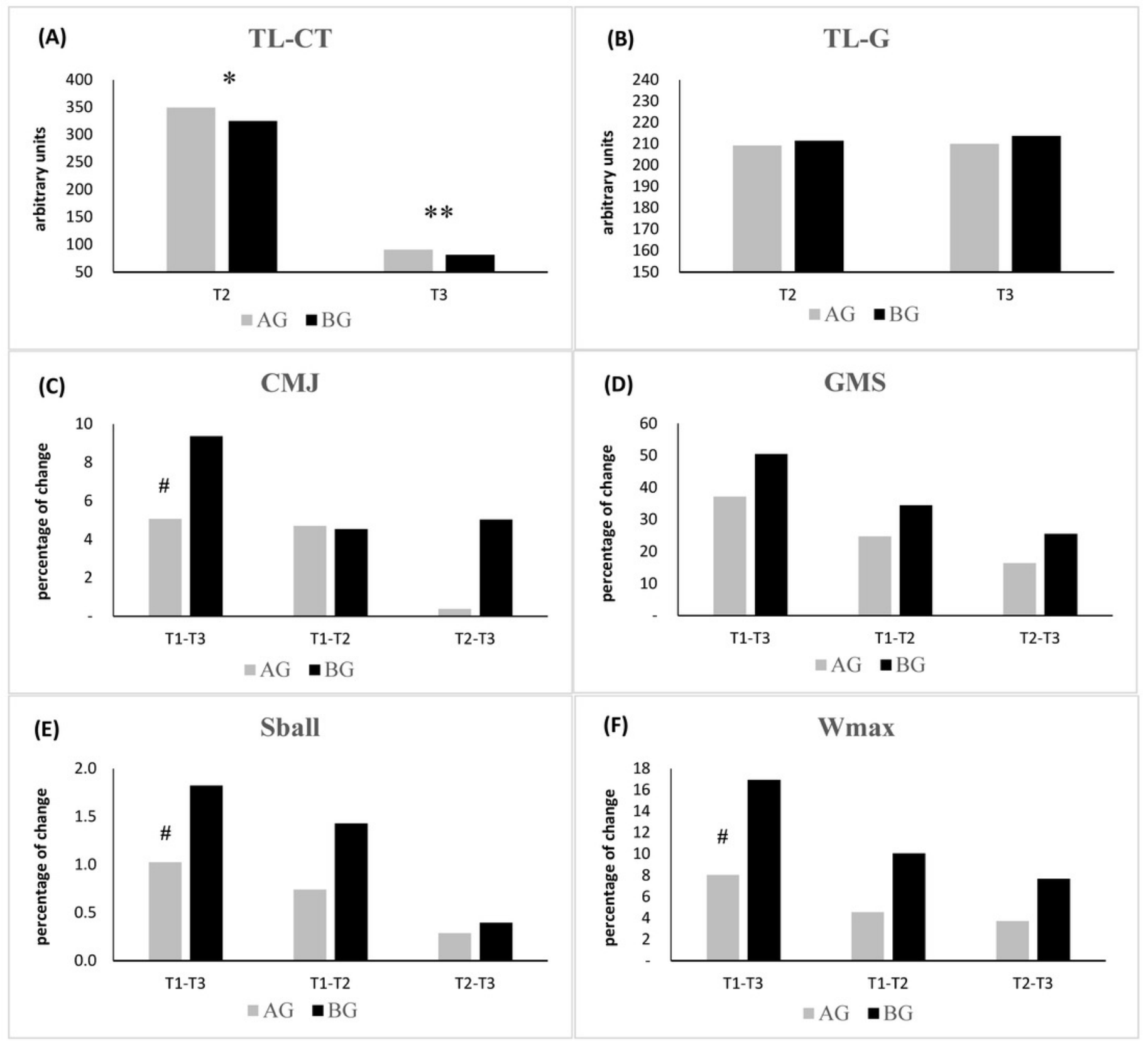

